AperTO - Archivio Istituzionale Open Access dell'Università di Torino

\title{
Relativistic Response Of A Fermi Gas.
}

\section{This is the author's manuscript}

Original Citation:

Availability:

This version is available http://hdl.handle.net/2318/121218

since

Published version:

DOI:10.1088/0305-4616/7/5/003

Terms of use:

Open Access

Anyone can freely access the full text of works made available as "Open Access". Works made available under a Creative Commons license can be used according to the terms and conditions of said license. Use of all other works requires consent of the right holder (author or publisher) if not exempted from copyright protection by the applicable law. 
Relativistic response of a Fermi gas

This article has been downloaded from IOPscience. Please scroll down to see the full text article.

1981 J. Phys. G: Nucl. Phys. 7 L93

(http://iopscience.iop.org/0305-4616/7/5/003)

View the table of contents for this issue, or go to the journal homepage for more

Download details:

IP Address: 192.84.147.42

The article was downloaded on 29/08/2012 at $12: 15$

Please note that terms and conditions apply. 


\title{
LETTER TO THE EDITOR
}

\section{Relativistic response of a Fermi gas}

\author{
W M Alberico and A Molinari \\ Istituto di Fisica Teorica dell'Università di Torino, Torino, Italy and INFN, Sezione di \\ Torino, Torino, Italy
}

Received 5 January 1981, in final form 9 February 1981

\begin{abstract}
The relativistic response of an assembly of non-interacting nucleons is calculated and compared with the non-relativistic limit.
\end{abstract}

In this Letter we compare the fully relativistic response of an assembly of non-interacting nucleons to an electromagnetic probe with the non-relativistic one. The finite size of the nucleons is also taken into account.

The motivation for this work is to point out that in most cases relativistic kinematics reduce the cross section for deep inelastic electron scattering (quasi-elastic peak). Our result contrasts some statements found in the literature (Moniz et al 1971, Brieva and Dellafiore 1977) $\dagger$.

In the Born approximation the double differential cross section (with respect to the scattering angle $\theta$ and the final energy $\varepsilon^{\prime}$ of an electron scattered out of a nucleus) reads (Moniz 1969, Donnelly and Walecka 1975)

$$
\frac{\mathrm{d}^{2} \sigma}{\mathrm{d} \Omega \mathrm{d} \varepsilon^{\prime}}=\sigma_{\mathrm{M}}\left\{S_{\mathrm{L}}(q, \omega)+\left[\frac{1}{2}\left(q_{\lambda}^{2} / q^{2}\right)+\tan ^{2} \frac{1}{2} \theta\right] S_{\mathrm{T}}(q, \omega)\right\}
$$

where $\sigma_{M}$ is the Mott cross section, and $q_{\lambda}=q, \omega / c$ is the four-momentum transfer. Keeping the full relativistic nucleon electromagnetic vertex one gets for the longitudinal and transverse structure functions (Moniz et al 1971)

$$
\begin{array}{r}
S_{\mathrm{L}}(q, \omega)=\frac{V}{(2 \pi)^{3}} \int \mathrm{d} \boldsymbol{k} \delta[\hbar \omega-(E(\boldsymbol{q}+\boldsymbol{k})-E(\boldsymbol{k}))] \frac{\theta\left(k_{\mathrm{F}}-k\right) \theta\left(|\boldsymbol{q}+\boldsymbol{k}|-k_{\mathrm{F}}\right)}{E(\boldsymbol{k}) E(\boldsymbol{q}+\boldsymbol{k})} \\
\times\left(\frac{T_{2}\left(q_{\lambda}^{2}\right)}{M^{2} c^{4}}[E(\boldsymbol{k})-\hbar \omega(k / q) \cos \tau]^{2}-\left(q_{\lambda}^{2} / q^{2}\right) T_{1}\left(q_{\lambda}^{2}\right)\right) \\
S_{\mathrm{T}}(q, \omega)=\frac{2 V}{(2 \pi)^{3}} \int \mathrm{d} \boldsymbol{k} \delta[\hbar \omega-(E(\boldsymbol{q}+\boldsymbol{k})-E(\boldsymbol{k}))] \frac{\theta\left(k_{\mathrm{F}}-k\right) \theta\left(|\boldsymbol{q}+\boldsymbol{k}|-k_{\mathrm{F}}\right)}{E(\boldsymbol{k}) E(\boldsymbol{q}+\boldsymbol{k})} \\
\times\left(T_{1}\left(q_{\lambda}^{2}\right)+\frac{T_{2}\left(q_{\lambda}^{2}\right)}{2 M^{2} c^{4}}(\hbar c k)^{2} \sin ^{2} \tau\right)
\end{array}
$$

+ Unfortunately there is a wrong sign in the coefficient of $T_{2}\left(q_{\lambda}^{2}\right)$ in formula $(2 a)$ of Brieva and Dellafiore (1977): this may be the reason for the incorrect conclusion about relativistic effects contained in that paper. 
where $V$ is the volume enclosing the system, $M$ the nucleon mass and $\tau$ the angle between $q$ and $k$. In $(2 a)$ and $(2 b)$

$$
\begin{aligned}
& T_{1}\left(q_{\lambda}^{2}\right)=\frac{1}{2}\left(\hbar c q_{\lambda}\right)^{2} \sum_{i}\left(F_{1}^{i}\left(q_{\lambda}^{2}\right)+2 M c^{2} F_{2}^{i}\left(q_{\lambda}^{2}\right)\right)^{2} \\
& T_{2}\left(q_{\lambda}^{2}\right)=2 M^{2} c^{4} \sum_{i}\left[F_{1}^{i 2}\left(q_{\lambda}^{2}\right)+\left(\hbar c q_{\lambda}\right)^{2} F_{2}^{i 2}\left(q_{\lambda}^{2}\right)\right]
\end{aligned}
$$

where the sum goes over the proton and the neutron and

$$
\begin{aligned}
& F_{1}^{\mathrm{p}}(0)=1 \quad F_{1}^{\mathrm{n}}(0)=0 \\
& 2 M c^{2} F_{2}^{\mathrm{p}}(0)=1.79 \quad 2 M c^{2} F_{2}^{\mathrm{n}}(0)=-1.91 .
\end{aligned}
$$

The $q_{i}$ dependence of the form factors has been chosen as (Weber 1967)

$$
F\left(q_{\lambda}^{2}\right)=\frac{1}{\left(1+q_{\lambda}^{2} / \Lambda^{2}\right)^{2}}
$$

with $\Lambda^{2}=18.1 \mathrm{fm}^{-2}$.

For an homogeneous, infinite system of non-interacting relativistic (both above and below the Fermi surface) nucleons one has, for the longitudinal cross section,

$$
\begin{aligned}
\left(\frac{1}{\sigma_{\mathrm{M}}} \frac{\mathrm{d}^{2} \sigma}{\mathrm{d} \Omega \mathrm{d} \varepsilon^{\prime}}\right)_{\mathrm{L}} & \\
= & \frac{V k_{\mathrm{F}}^{2}}{4 \pi^{2} \hbar c q}\left(\frac { T _ { 2 } ( q _ { \lambda } ^ { 2 } ) } { 2 M ^ { 2 } c ^ { 4 } } ( 1 - \frac { \alpha ^ { 2 } \nu ^ { 2 } } { q ^ { 2 } } ) ^ { 2 } \left\{\frac { 2 } { 3 } \left[\left(\frac{1}{\alpha^{2}}+1\right)^{3 / 2}\right.\right.\right. \\
& \left.\left.-\left(\frac{1}{\alpha^{2}}+q_{0}^{2}\right)^{3 / 2}\right]+\alpha \nu\left(1-q_{0}^{2}\right)+\frac{1}{2} \alpha^{2} \nu^{2}\left[\left(\frac{1}{\alpha^{2}}+1\right)^{1 / 2}-\left(\frac{1}{\alpha^{2}}+q_{0}^{2}\right)^{1 / 2}\right]\right\} \\
& \left.-\left(1-\frac{\alpha^{2} \nu^{2}}{q^{2}}\right) \frac{T_{1}\left(q_{\lambda}^{2}\right)}{\left(\hbar c k_{\mathrm{F}}\right)^{2}}\left[\left(\frac{1}{\alpha^{2}}+1\right)^{1 / 2}-\left(\frac{1}{\alpha^{2}}+q_{0}^{2}\right)^{1 / 2}\right]\right)
\end{aligned}
$$

and, for the transverse cross section,

$$
\begin{aligned}
\left(\frac{1}{\sigma_{\mathrm{M}}} \frac{\mathrm{d}^{2} \sigma}{\mathrm{d} \Omega \mathrm{d} \varepsilon^{\prime}}\right)_{\mathrm{T}} & \\
= & \frac{V k_{\mathrm{F}}^{2}}{2 \pi^{2} \hbar c q}\left[\frac{1}{2}\left(1-\frac{\alpha^{2} \nu^{2}}{q^{2}}\right)+\tan ^{2} \frac{1}{2} \theta\right] \\
& \times\left(\frac{T_{1}\left(q_{\lambda}^{2}\right)}{\left(\hbar c k_{\mathrm{F}}\right)^{2}}\left[\left(\frac{1}{\alpha^{2}}+1\right)^{1 / 2}-\left(\frac{1}{\alpha^{2}}+q_{0}^{2}\right)^{1 / 2}\right]+\frac{T_{2}\left(q_{\lambda}^{2}\right)}{2 M^{2} c^{4}}\right. \\
& \times\left\{\left(\frac{1}{\alpha^{2}}+1\right)^{1 / 2}-q_{0}^{2}\left(\frac{1}{\alpha^{2}}+q_{0}^{2}\right)^{1 / 2}-\frac{2}{3}\left(1+\frac{\alpha^{2} \nu^{2}}{2 q^{2}}\right)\left[\left(\frac{1}{\alpha^{2}}+1\right)^{3 / 2}\right.\right. \\
& \left.-\left(\frac{1}{\alpha^{2}}+q_{0}^{2}\right)^{3 / 2}\right]+\frac{\alpha \nu}{2}\left(1-\frac{\alpha^{2} \nu^{2}}{q^{2}}\right)\left(1-q_{0}^{2}\right)-\frac{q^{2}}{4}\left(1-\frac{\alpha^{2} \nu^{2}}{q^{2}}\right)^{2} \\
& \left.\left.\times\left[\left(\frac{1}{\alpha^{2}}+1\right)^{1 / 2}-\left(\frac{1}{\alpha^{2}}+q_{0}^{2}\right)^{1 / 2}\right]\right\}\right)
\end{aligned}
$$


where $q, \nu$ are the dimensionless momentum and energy transfer ( $q$ in units of the Fermi momentum $k_{\mathrm{F}}$ and $\left.\nu=M \omega / \hbar k_{\mathrm{F}}^{2}\right), \alpha=\hbar k_{\mathrm{F}} / M c$ and

$$
q_{0}=\frac{\nu}{q}\left(\frac{1}{1-\alpha^{2} \nu^{2} / q^{2}}+\frac{\alpha^{2} q^{2}}{4}\right)^{1 / 2}-\frac{q}{2} .
$$

Formulae (4) and (5) hold in the regions of the $(\nu, q)$ plane defined by $q \geqslant 2$ and

$$
\left\{\left[1+\alpha^{2}(1+q)^{2}\right]^{1 / 2}-\left(1+\alpha^{2}\right)^{1 / 2}\right\} / \alpha^{2} \geqslant \nu \geqslant\left\{\left[1+\alpha^{2}(q-1)^{2}\right]^{1 / 2}-\left(1+\alpha^{2}\right)^{1 / 2}\right\} / \alpha^{2}
$$

where relativistic effects are expected to be sizable, or $q<2$ and

$$
\left\{\left[1+\alpha^{2}(1+q)^{2}\right]^{1 / 2}-\left(1+\alpha^{2}\right)^{1 / 2}\right\} / \alpha^{2} \geqslant \nu \geqslant\left\{\left(1+\alpha^{2}\right)^{1 / 2}-\left[1+\alpha^{2}(1-q)^{2}\right]^{1 / 2}\right\} / \alpha^{2} .
$$

To appreciate the role of relativity in the nuclear response we expand (4) and (5) in powers of $\alpha^{2}$. Keeping only the first two terms of the expansion one gets
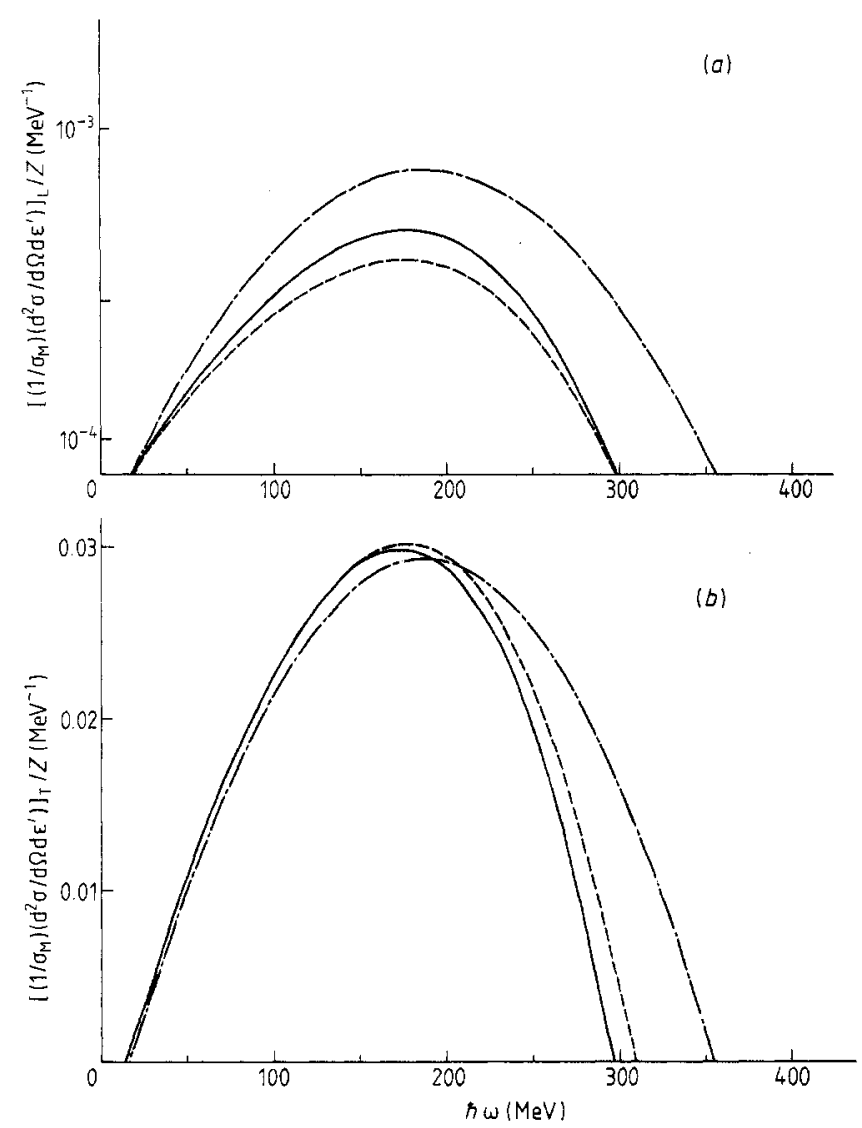

Figure 1. The longitudinal (a) and transverse at $\theta=150^{\circ}(b)$ double differential cross sections, in the Born approximation, as a function of the transferred energy at $q=3 \mathrm{fm}^{-1}$. The full curves correspond to the relativistic calculation, the chain curves to the nonrelativistic one and the broken curves to formula (8). The finite size of nucleons is taken into account; $k_{\mathrm{F}}$ is $1.36 \mathrm{fm}^{-1}$. 


$$
\begin{aligned}
\left(\frac{1}{\sigma_{\mathrm{M}}} \frac{\mathrm{d}^{2} \sigma}{\mathrm{d} \Omega \mathrm{d} \varepsilon^{\prime}}\right)_{\mathrm{L}} & \left\{-\frac{V}{\pi} \operatorname{Im} \Pi^{0}(q, \nu)\left[1+\frac{1}{2} \alpha^{2}\left(\nu+\frac{1}{2}\left(1+k_{0}^{2}\right)-2\left(\nu^{2} / q^{2}\right)\right.\right.\right. \\
\simeq & \left.\left.\left.-2.29 q^{2}+8 \frac{k_{\mathrm{F}}^{2} \nu^{2}}{\Lambda^{2}+k_{\mathrm{F}}^{2} q^{2}}\right)\right]-\frac{V M k_{\mathrm{F}}}{(2 \pi \hbar)^{2}} \alpha^{2}(1+\nu) \frac{\nu k_{0}}{q^{2}}\right\}\left(1+\frac{k_{\mathrm{F}}^{2} q^{2}}{\Lambda^{2}}\right)^{-4} \\
\left(\frac{1}{\sigma_{\mathrm{M}}} \frac{\mathrm{d}^{2} \sigma}{\mathrm{d} \Omega \mathrm{d} \varepsilon^{\prime}}\right)_{\mathrm{T}} & \\
\simeq & \left\{-\frac{V}{\pi} \operatorname{Im} \Pi^{0}(q, \nu) \alpha^{2}\left(\frac{1}{2}+\tan ^{2} \frac{1}{2} \theta\right)\left[\left[11.43 \times \frac{1}{2} q^{2}+\frac{1}{2}\left(1-k_{0}^{2}\right)\right]\right.\right. \\
& \times\left(1+\frac{4 \alpha^{2} k_{\mathrm{F}}^{2} \nu^{2}}{\Lambda^{2}+k_{\mathrm{F}}^{2} q^{2}}\right)-\frac{1}{2} \alpha^{2}\left\{10.43\left[\frac{1}{4} q^{2}\left(1+k_{0}^{2}\right)+\nu^{2}\right]-6.85 \times \frac{1}{4} q^{2}\left(1-k_{0}^{2}\right)\right.
\end{aligned}
$$

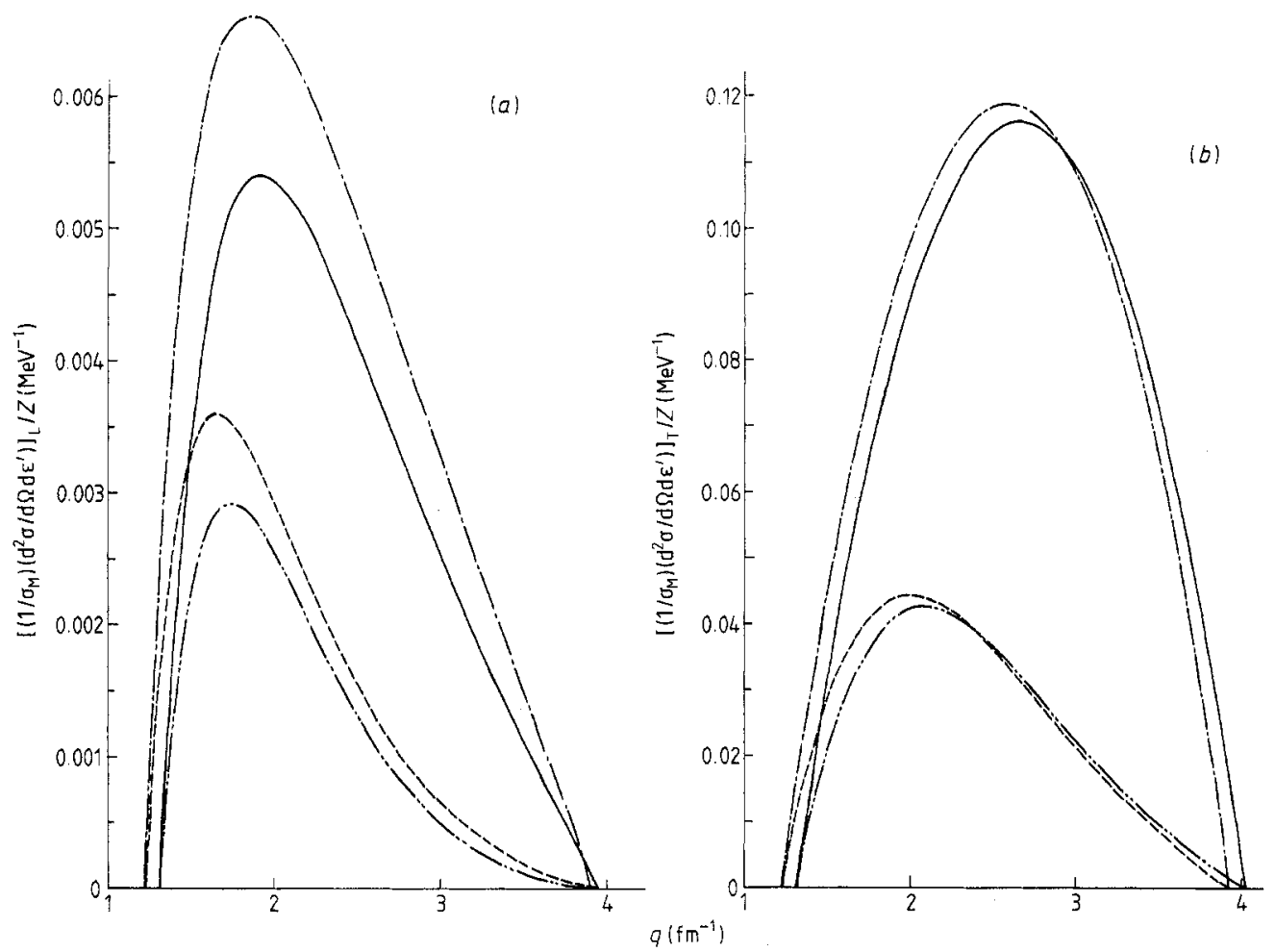

Figure 2. The longitudinal $(a)$ and transverse at $\theta=150^{\circ}(b)$ double differential cross sections, in the Born approximation, as a function of the transferred momentum $q$ at fixed energy $\hbar \omega=100 \mathrm{MeV}$. The full curves correspond to the relativistic calculation and the chain curves to the non-relativistic one for point-like nucleons; the double chain and broken curves are, respectively, the relativistic and non-relativistic response from extended nucleons; $k_{\mathrm{F}}$ is $1.36 \mathrm{fm}^{-1}$. 


$$
\begin{aligned}
& \left.\left.+2 \frac{\nu^{3}}{q^{2}}+\frac{\nu^{2}}{2 q^{2}}\left(1-\frac{\nu^{2}}{q^{2}}\right)+\frac{1}{8} \nu\left(q^{2}-\nu\right)+\frac{1}{3}\left(1+k_{0}^{2}+k_{0}^{4}\right)\right\}\right] \\
& -\alpha^{4} \frac{V M k_{\mathrm{F}}}{(2 \pi \hbar)^{2}} \frac{\nu}{2 q^{2}}\left(11.43 q^{2}+1-k_{0}^{2}\right)\left[\frac{\nu}{2 q}\left(1-k_{0}^{2}\right)+\left(\frac{1}{2}+\tan ^{2} \frac{1}{2} \theta\right)\right. \\
& \left.\left.\times\left(\frac{\nu^{2}}{q^{2}}+\frac{q^{2}}{4}\right) k_{0}\right]\right\}\left(1+\frac{k_{\mathrm{F}}^{2} q^{2}}{\Lambda^{2}}\right)^{-4}
\end{aligned}
$$

where $k_{0}=\nu / q-\frac{1}{2} q$ and $\Pi^{0}(q, \nu)$ is the time-ordered density-density correlation function of a free Fermi gas:

$$
\operatorname{Im} \Pi^{0}(q, \nu)=-\frac{M k_{\mathrm{F}}}{4 \pi \hbar^{2}} \frac{1}{q}\left[1-\left(\frac{\nu}{q}-\frac{q}{2}\right)^{2}\right] \quad(q \geqslant 2) .
$$

Thus, to leading order, one recovers the familiar result of linear response theory. Numerically (8) and (9) are very close to (4) and (5) as the expansion parameter $\alpha^{2}$ is small.

In figure 1 $(a)$ the longitudinal cross section is plotted as a function of the energy for $q=3 \mathrm{fm}^{-1}$. The shrinking effect associated with relativistic kinematics is clearly exhibited, particularly at large electron energy loss.

The transverse cross section is shown in figure $1(b)$, where the same effect is seen to occur for large $\omega$. Note that at $\theta=150^{\circ}$ the transverse cross section is about 20 times as large as the longitudinal one.

For smaller values of $\omega$ the reduction of the cross section due to relativistic kinematics takes place, essentially, only in the longitudinal case and for momentum transfers which are not too large.

The corresponding situation is illustrated in figures $2(a)$ and $2(b)$, where the cross sections are displayed as a function of $q$ for $\hbar \omega=100 \mathrm{MeV}$.

Perhaps it is worthwhile to recall that a much stronger reduction in the nuclear response is linked with the finite size of the nucleon which, furthermore, substantially reshapes the cross section: indeed for large momenta the exchanged virtual photon has a wavelength $\lambda$ significantly shorter than the root-mean-square proton radius $(\sim 0.8 \mathrm{fm})$. This result is, of course, known, but we felt it worthwhile to present it again in conjunction with the relativistic effects.

We believe that these features will qualitatively persist when the interaction among the nucleons is switched on.

We wish to thank Dr R Cenni for discussions.

\section{References}

Brieva F A and Dellafiore A 1977 Nucl. Phys. A 292445

Donnelly T W and Walecka J D 1975 Ann. Rev. Nucl. Sci. 25329

Moniz E J 1969 Phys. Rev. 1841154

Moniz E J et al 1971 Phys, Rev. Lett. 26446

Weber G 1967 Proc. Symp. on Electron and Photon Interactions, Stanford p 59 